\title{
The value of medical pre-operative assessment for patients receiving penile prostheses
}

\author{
Alex Tatem ${ }^{1}$, Jason R. Kovac ${ }^{2}$ \\ ${ }^{1}$ Department of Urology, Indiana University, Indianapolis, Indiana, USA; ${ }^{2}$ Men's Health Center, Indianapolis, Indiana, USA \\ Correspondence to: Dr. Jason R. Kovac, MD, PhD, FACS, FRCSC. Men's Health Center, 8240 Naab Road, Suite 220, Indianapolis, Indiana 46260, \\ USA. Email: jkovac@urologyin.com \\ Comment on: Masterson TA, Palmer J, Dubin J, et al. Medical pre-operative considerations for patients undergoing penile implantation. Transl Androl \\ Urol 2017;6:S824-9.
}

Submitted Oct 30, 2017. Accepted for publication Nov 01, 2017.

doi: $10.21037 / \operatorname{tau} .2017 .11 .16$

View this article at: http://dx.doi.org/10.21037/tau.2017.11.16

Penile prosthesis placement is both safe and efficacious for the treatment of medically refractory erectile dysfunction (ED). Nevertheless, as with all surgery, it carries with it the potential for complications. Consequently, both proper patient selection and risk management are essential for prosthetic surgeons. While very few patients are free of major comorbidities, others have multiple medical issues. Causes of ED include both systemic medical conditions such as diabetes, cardiac disease, hypertension and obesity as well as specific causes such as post surgical prostate, bladder and rectal cancer patients. As such, optimization prior to surgery could have multiple benefits to the prosthetic surgeon.

Pre-operative testing specific to penile prosthesis surgeries includes the routine assessment of urine cultures, hemoglobin A1c (1) and cystoscopy in patients with potential for urethral/bladder neck issues. In routine surgical patients, universal routine testing of full blood count, electrolytes and pulmonary function tests are not cost-effective (2); however, men with ED typically exhibit more extensive issues. In general, pre-operative assessment has yielded improved patient satisfaction (3) as well as reductions in redundant medical tests and inhospital consultations $(4,5)$. Particular value can be found in the identification of new medical problems that are more likely to result in surgical delays or cancellations (6). Indeed, hospitals benefit from such early assessment and optimization since decreases in last minute operating room cancellations significantly reduces financial impacts (7).

At our institution, we routinely send all patients for pre- admission evaluation and consultation prior to surgery. During the course of the past few years, we have had numerous issues identified at the pre-operative stage of surgical planning including uncontrolled hypertension from recent medication changes, optimization of uncontrolled anti-coagulation medications, urinary and bladder infections as well as urethral strictures and bladder neck contractures. No specific studies exist to assess the value of pre-operative assessment in men with ED undergoing penile prosthesis surgery. A multi-center trial would no doubt be of value.

\section{Acknowledgements}

None.

\section{Footnote}

Conflicts of Interest: The authors have no conflicts of interest to declare.

\section{References}

1. Masterson TA, Palmer J, Dubin J, et al. Medical preoperative considerations for patients undergoing penile implantation. Transl Androl Urol 2017;6:S824-9.

2. Czoski-Murray C, Lloyd Jones M, McCabe C, et al. What is the value of routinely testing full blood count, electrolytes and urea, and pulmonary function tests before elective surgery in patients with no apparent clinical indication and in subgroups of patients with common 
comorbidities: a systematic review of the clinical and cost-effective literature. Health Technol Assess 2012;16:i-xvi, 1-159.

3. Hepner DL, Bader AM, Hurwitz S, et al. Patient satisfaction with preoperative assessment in a preoperative assessment testing clinic. Anesth Analg 2004;98:1099-105.

4. Fischer SP. Development and effectiveness of an anesthesia preoperative evaluation clinic in a teaching hospital. Anesthesiology 1996;85:196-206.

5. Tsen LC, Segal S, Pothier M, et al. The effect of

Cite this article as: Tatem A, Kovac JR. The value of medical pre-operative assessment for patients receiving penile prostheses. Transl Androl Urol 2017;6(Suppl 5):S830-S831. doi: $10.21037 /$ tau.2017.11.16 alterations in a preoperative assessment clinic on reducing the number and improving the yield of cardiology consultations. Anesth Analg 2002;95:1563-8.

6. Correll DJ, Bader AM, Hull MW, et al. Value of preoperative clinic visits in identifying issues with potential impact on operating room efficiency. Anesthesiology 2006;105:1254-9; discussion 6A.

7. Ferschl MB, Tung A, Sweitzer B, et al. Preoperative clinic visits reduce operating room cancellations and delays. Anesthesiology 2005;103:855-9. 